\title{
SOURCE IDENTIFICATION AND MITIGATION OF INDUSTRIAL POLLUTION
}

\author{
Eva Eriksson ${ }^{I}$ \\ Katarina Hansson ${ }^{2}$ \\ Anna Ledin ${ }^{1,3}$ \\ ${ }^{I}$ Technical University of Denmark, Denmark, \\ ${ }^{2}$ Environment and social structure, Eslöv municipality, Sweden \\ Linköping University, Sweden
}

\begin{abstract}
Diffuse pollution comprises of pollution arising from a variety of dispersed, of ten minor, point sources. The aim of this study was to identify which hazardous substances that are discharged to the wastewater treatment plant (WWTP) in Eslöv municipality from industries and businesses. Based on these findings control actions was suggested in order to improve the quality of the sludge produced in the WWTP. Here, 43 industries and businesses connected to the municipal sewer system in Eslöv municipality reported commodities and activitiesa industrial process and maintenance products, cleaning detergents and other chemicals discharged to the sewer system, including composition of individual substances. Over 900 commodities and 2300 substances were recorded. Of these were 92 substances frequently used (present in $\geq 5$ commodities). Most prominently was iso-propanol which was found in 61 commodities. A hazard identification and ranking of 346 substances showed that $9 \%$ of the substances pose a potential hazard in the sludge. Seven of the most frequently used substances consisted of petroleum based hydrocarbons. Most of the industries and businesses referred to having an oil separator installed on the premises. However, recent studies have shown that effluents from oil separators in many cases have a hydrocarbon oil index $>50 \mathrm{mg} / \mathrm{L}$, which indicates that the efficiency should be questioned. Among the pesticides and preservatives identified were isothiazolines, Bronopol and several parabens. The isothiazolines and Bronopol originated from industrial applications, whereas the parabens derived from the personal care products used for staff wellbeing. Noteworthy is that both the isothiazolines and Bronopol were present in different brands of car shampoo. Generally, this study was hampered by incomplete data provided by the industries and businesses as well as the lack of inherent data, primarily biodegradation, used in the hazard identification and ranking of the organic substances.
\end{abstract}

\section{KEYWORDS}

Diffuse pollution: Hazard identification: Source control: Source screening

\section{INTRODUCTION}

Diffuse pollution comprises of pollution arising from a variety of dispersed. often minor, point sources. Although they as individuals often are considered as minor contributors, their collective contribution may be a significant sourcea No single solution to tackle diffuse pollution has been put forward. Among reliable measures are changes in the handling and 
house keeping of substances, in order to reduce impacts from diffuse pollution. For example as a waste discharge charge system (WDCS) in South Africa [1]. In Sweden, some municipalities, the farmersò organisations (LRF), the food industry, the commercial trade organization and non-governmental environmental and consumer focussed organisations are collaborating in a project called ReVAQ, with the purpose to enhance the quality of municipal sludge to be applied on agricultural land in a sustainable manor [2]. Sludge is not used on agricultural land today due to the fear for pollution of the soil and consequently pollution of the crops. One of the participants, Eslöv municipality, located in the south of Sweden, aims to mitigate hazardous pollutants from their sludge and treated wastewater. The focus, has up to now, been on heavy metals (with a special emphasis on cadmium) and PBT (persistent, bioaccumulating and toxic) organic substances.

The aim of this study was to identify which hazardous substances that are discharged to the wastewater treatment plant in Eslöv municipality, and to clarify from which industries and businesses these are emitted. Based on these findings, further control actions will be suggested. Possible actions are, e.g.. implementation of source control options, such as changing one commodity into another and thereby reducing the discharges of a non-wanted substance, or changing the handling strategy, e.g.. collect spills and treat them as solid waste instead of discharge them to the sewer systems. Other possibilities are implementation of local treatment at the industry/business.

\section{MATERIALS AND METHODS}

The project started by launching an information campaign including a questionnaire to industries and businesses connected to the municipal sewer system in Eslöv, see Figure 1.

The questionnaire included an Excel sheet in which the businesses were asked to report trade name and chemical composition of the commodities used in their operation and maintenance. They should also report CAS registry numbers for individual substances included in the commodities as well as estimation on how much that is discharged into the sewer system in $\mathrm{kg}$ or litres on an annual basis. Additionally, any risk phrases and ecotoxicity data listed on the commodities" Material safety data sheet (MSDS) should also be included.

The reported substances were subjected to a hazard identification according the RICH-tool (ranking and identification of chemicals hazards) procedure described by Baun et al. [3], where the substances are ranked based on their inherent properties (solubility, sorption, evaporation), effects (acute and long-term toxicity) and environmental fate (degradation and bioaccumulation). The methodology consists of a decision tree in which hazardous and problematic compounds are identified. To visualize the sorting of XOC the decision tree can be described as a funnel fitted with several filters. The filters have been set according to specified criteria based on sorption, volatility, persistence to biodegradation, potential for bioaccumulation and aquatic toxicity. There are also one on/off filter for technical/aesthetical problems and a long-term chronic effects-filter considering cancer, mutagenic and reproduction hazards, endocrine disruption effects and allergenic effects. The output is a classification of the compounds in three categories (white, grey and black) depending on their priority as possible pollutants. White compounds are considered as non-hazards, which mean that these compounds will be excluded from the continuing evaluation with CHIAT. Grey compounds are passed on to the next filter. These compounds may or may not be potential hazards depending on the outcome of the following filtration. Black compounds are considered as potential hazards. 
Primarily inherent data collected in a project focussing on domestic wastewater were, due to the limited time frame. used for the ranking procedure [4]. Since the industrial commodities and substances generally are not present in this waste flow, toxicity data originating from the MSDS were applied and supported by inherent data on sorption and volatilization based on measured data (PhysProp) and estimated data (EPI Suite) [5].

Commodities containing substances identified as hazardous were in the next step evaluated in order to find possible actions, e.g.. implementation of source control options.

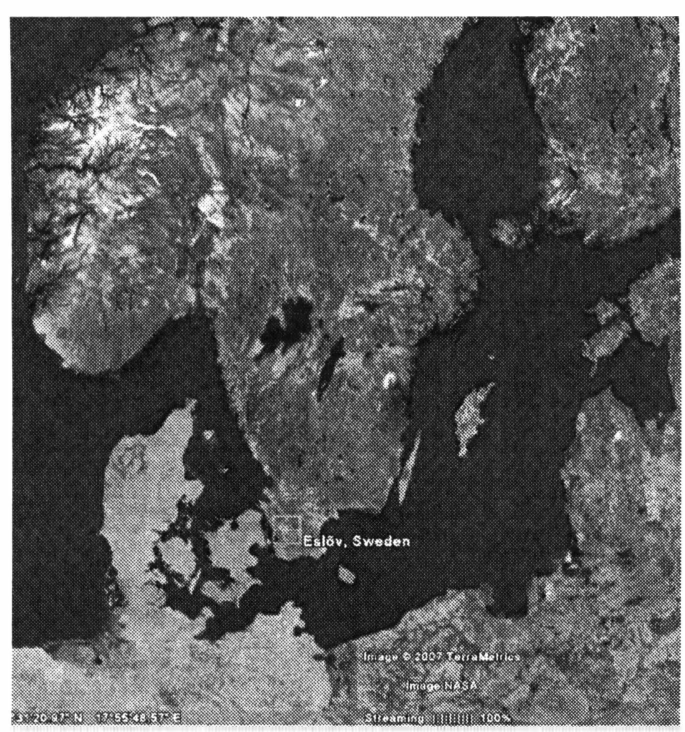

Figure 1. Location of Eslöv municipality in the southern part of Sweden (from Google Earth 2007).

\section{RESULTS AND DISCUSSION}

Feedback was obtained from 42 industries/businesses in the municipality of Eslöv. These range from food industry to a cemetery administration, with a high representation of car and farming machines styling and maintenance. Nine hundred and one commodities have been reported to be in use. These include process and industry chemicals; equipment and office cleaning detergents; laundry detergents and personal care products. These commodities were reported to contain at least 2382 substances of which 2007 were reported with CAS and 375 were reported by name. The actual number of substances (1091) is; however, lower as gross number includes duplicates, i.e. present in more then one commodity.

The highly prioritized substance cadmium $(\mathrm{Cd})$ was found not to be reported by any of the industries and businesses participating in this study. In a previous study five industries and businesses had $\mathrm{Cd}$ concentrations $>1 \mathrm{mg} / \mathrm{L}$ in their wastewater [2] indicating that $\mathrm{Cd}$ may originate from indirect sources such as tear and wear of industrial appliances and vehicles. 


\subsection{Frequently present substances}

A total of 92 substances were present in 5 or more commodities, see Table 1. Most frequently present were iso-propanol, butane and propane which were found in 61, 42 and 46 commodities, respectively. Thereafter were the surfactants alcohol ethoxylates and the solvent acetone present in 35 and 31 commodities. Ten substances were found in 20-29 commodities, 21 in the range of 10-19 and a total of 55 were present in 5-9 commodities.

Other substances as for example, the Bisphenol A derivate Bisphenol-A-diglycidether was present in 5 commodities related to mending paste and floor paint. Three silicates (Sodium silicate, Disodium metasilicate and Sodium metasilicate pentahydrate) were also found among the most frequently used substances. These were present in various cleaning and surface treatment commodities such as degreasing products, vehicle wax, vehicle shampoos, protective cream, cleaning detergent and carousel cleaners.

\subsection{Inorganic acids and bases}

Phosphoric acid, hydrochloric acid and sulphuric acid were present in 17,6 and 5 commodities, respectively. Using the substance presence in commodities $(\%)$, the volume used by each business and the business estimated discharge to wastewater (\%) it was found that on an annual basis $1.1-1.7 \mathrm{~m}^{3}$ of phosphoric acid, $1.9 \mathrm{~m}^{3}$ of hydrochloric acid (of which $98.8 \%$ is reported to neutralized or protolyzed before discharge) and $0.3-0.7 \mathrm{~m}^{3}$ sulphuric acid are entering the wastewater.

Similarly for the bases it was found that sodium hydroxide and potassium hydroxide were present in 29 and 18 commodities, respectively. It was calculated, from the reported annual use of various commodities, that at least 15.2 tons of sodium hydroxide and 0.4-1.1 tons of potassium hydroxide are discharge to Eslöv WWTP annually. Their applications were primarily cleaning, de-greasing and ion exchange. 
Table 1. Substances present in five or more commodities.

Number of commodities
Organic and inorganic substances

\begin{tabular}{ll}
\hline$>60$ & Iso-propanol \\
\hline $40-49$ & Butane, Propane \\
\hline $30-39$ & Acetone, Alcohol ethoxylates, Water \\
\hline $30-29$ & $\begin{array}{l}\text { Cumene hydroperoxide. Dipropylene glycol methyl ether, Disodium metasilicate, } \\
\text { Distillate(petroleum) hydrotreated light, Ethanol, Ethyl acetate, Hydrotreated heavy } \\
\text { naphtha, Nitrilotriacetic acid, Trisodium salt sodium hydroxide, Xylenes }\end{array}$ \\
\hline & $\begin{array}{l}\text { 2-(2-Butoxyethoxy)ethanol. 2-Butoxy ethanol, Butyl acetate, Carbon Dioxide, } \\
\text { Ethanol Amine, Ethyl 2-cyanoacrylate, Ethylene Glycol, Fatty alcohol polyglycol } \\
\text { ether, Fisherbrand vacuum pump oil, Hydrodesulfurized heavy naphtha, Methyl } \\
\text { ethyl ketone, N-(2-carboxyethyl)-N-(2-ethylhexyl)beta-alanine monosodium salt, } \\
\text { Naphtha, petroleum hydrotreated light. Neodol 1-9, Phosphoric acid, Poly(oxy-1,2- } \\
\text { ethanediyl), alpha-tridecyl-omega-hydroxy- branched, Potassium Diphosphate, } \\
\text { Potassium hydroxide, Propylene Glycol Monomethyl Ether, Sodium metasilicate } \\
\text { pentahydrate, Toluene }\end{array}$ \\
\hline
\end{tabular}

1,1,1.2-Tetrafluoroethane, 1-Acetyl-2-phenylhydrazine, 1-Decene, homopolymer hydrogenated, 2-(2-Ethoxyethoxy)ethanol, 2.4,7,9-Tetramethyl-5-decyn-4,7-diol, 2-Bromo-2-nitropropane-1.3-diol, 2-Ethylhexanolethoxylate, 2-Hydroxyethyl methacrylate, 2-Hydroxypropyl methacrylate, 2-Methyl-4-Isothiazolin-3-one. 5Chloro-2-methyl-4-isothiazolin-3-one. Ammonium hydroxide, Benzoyl peroxide, Bis(2-hydroxyethyl)coco alkylamine acetate, Bisphenol-A-diglycidether. Cellulose gum (Edifas B), Citric acid, Citric acid monohydrate, Cocamidopropyl betaine, Cyclohexane, Ethoxylated propoxylated alcohols C12-14, Ethyl benzene, Ethyl methyl ketone oxime, Ethyltriacetoxysilane, Glycine, N-[2(carboxymethoxy)ethyl]-N-[2-[(1-oxodecyl)amino]ethyl]- disodium salt, Glycerine, 5-9 Hydrochloric acid, Limestone, Methacrylic acid. Methyl Ether, Methyl methacrylate, Methylene diphenyl diisocyanate, Methyltriacetoxysilane, N,NDiethyl-p-toluidine, N,N-Dimethyl-o-toluidine, Naphtha (petroleum), hydrodesulfurized light dearomatized, Nitrilotriacetic acid trisodium salt monohydrate, Oxo C13 alcohol ethoxylate, Paraffins (petroleum), normal C5-20, Polyethoxylated-cis-9-octadecenylamine, Polyethylene glycol monooleate, Polymethylene polyphenyl isocyanate

Propanol oxybis- dibenzoate, Propylene glycol monomethyl ether acetate, Quaternary ammonium compounds coco alkylbis(hydroxyethyl)methyl ethoxylated Me sulfates (salts), Sodium alkyl ether sulphate, Sodium carbonate. Sodium chloride. Sodium percarbonate. Sodium silicate, Sodium tripolyphosphate, Sulphuric acid. Titanium dioxide, Triethanolamine. Zinc oxide

Additionally, both some acids and based were reported to be neutralised with Magnodol (dolomite rock) before discharge, but no information on how much Magnodol that are used on an annual basis was reported. Other $\mathrm{pH}$ affecting inorganic substances that are among the most frequently used are ammonium hydroxide, sodium carbonate and limestone present in 7 , 7 and 5 commodities. respectively. 


\subsection{BETXs (benzene, ethyl benzene, toluene and xylenes)}

Several of the aromatic organic solvents, the BETXs. were found among the frequently used substances ethyl benzene, toluene and xylenes. in 5, 12 and 25 commodities, respectively. Benzene was present in only two commodities and reported in the concentration of $<1 \%$. Benzene is a priority substance in the field of water policy [6] and as such it is subjected to progressive reduction of discharges, emissions and losses [7].

\subsection{Oil and grease}

Many of the participating industries and businesses were connected with operation and maintained of vehicles with combustion engines or vehicle parts, and consequently there is a strong correlation with the lubricants used. Seven of the most frequently used substances were petroleum based oil or grease related substances; Distillate (petroleum) hydrotreated light present in 29 commodities, Hydrotreated heavy naphtha (28). Naphtha petroleum hydrotreated light (17), Hydrodesulfurized heavy naphtha (15), Fisherbrand vacuum pump oil (11), and Paraffins (petroleum) normal C5-20 (9) as well as Naphtha (petroleum) hydrodesulfurized light dearomatized (7).

Several of the businesses referred to having an oil separator installed. A measuring campaigned was carried out, within the ReVAQ project in 2003. Here. the wastewater composition from 26 industries/businesses after treatment in the oil separators were investigated and the results showed that $12 \%$ of the industries/businesses had an oil index $>50$ $\mathrm{mg} / \mathrm{L}$ af ter the oil separator [2]. Thus, a careful evaluation of the efficiency of oil separators is needed for the oil and grease using industries/businesses.

\subsection{Pesticides and preservatives}

Three pesticides were among the most frequently used substances. These were 2-Bromo-2nitropropane-1,3-diol (Bronopol) which was present in 5 commodities and two isothiazolines (2-Methyl-4-isothiazolin-3-one and 5-Chloro-2-methyl-4-isothiazolin-3-one) both present in 9 commodities. Bronopol was found to be present in wall paint, boiler water and car shampoo whereas the isothiazolines were present in wall and floor paints, glue for wood applications, car shampoo and sanitary cleaning products. The applicability for using pesticides in car shampoo is unclear. Other isothiazolines and methyl and propyl parabens (personal care products preservatives) were used in less than five each. No agricultural or garden related facilities participated in this study and accordingly no herbicides were found. Though it cannot be excluded that the food industry indirectly may contribute with herbicides from processing of vegetable materials containing herbicide residues.

\subsection{Hazard identification by RICH ranking}

A total of 346 organic substances were selected to be subjected to the RICH-tool ranking, corresponding to those with CAS included and inherent data availablea It was noted that 69 lacked some inherent data making them not possible to fully evaluate. Of the remaining were 218 listed as non-hazards ("white"), 3 as possible hazards ("grey") and 53 as potential hazards ("black") in the sludge phase. In the aqueous/dissolved phase were 241 ranked as "white", 4 as "grey" and 37 as "black", see Figure 2.

Some of the "black" substances overlapped between the solid/sludge and the aqueous phase. thus a total of 76 substances were all in all identified as potential hazards. Among the "black" substances were three isothiazolines, one phthalate, the medium-chained chlorinated paraffins 
C14-17, two different salts of the linear alkyl benzene sulfonates (LAS), two red dyes and two iso-cyanates as well as two diiso-cyanates. Nine of the "black" substances are also listed as "Priority risk reduction substances" or "Phase-out substances" by the Swedish Chemicals Agency [8].
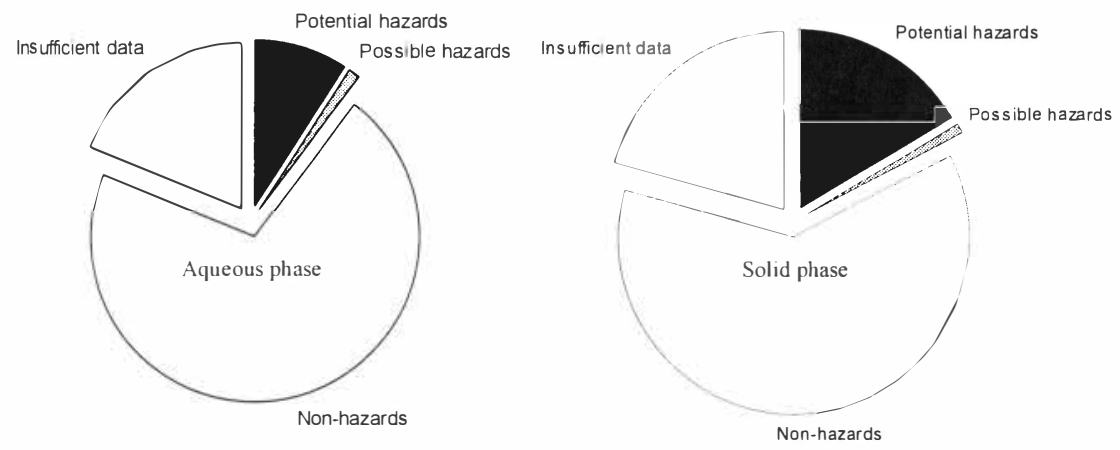

Figure 2. Outcome of the hazard identification and ranking procedure.

\subsection{Mitigation}

Procedures for handling of petroleum based hydrocarbons and an efficiently review of the oil separators is suggested based on the findings presented above. Also, a discussion of substitution to rape methyl ester (RME) used by one industry as well as other bio-fuels and lubricates is recommended. The use of car shampoo containing pesticides in questionable both with respect to applicability and further dispersal in the environment and should be discussed with the businesses in question.

Most of the industries and businesses reported to use eco-labelled cleaning detergents but some commodities were found to contain linear alkylbenzene sulphonates (LAS) which has been discussed in the scientific community due to its low biodegradabitity during anaerobic conditions and toxicity to anaerobic micro-organisms $[9,10,11]$.

The hazard identification showed that the chlorinated paraffins (C14-17) may pose a potential hazard to sludge and subsequently sludge amended soil. These are closely related to the short chain chlorinated paraffins (SCCP: C10-13) which are classified as priority hazardous substances [6] and as such are subjected to the cessation or phasing-out of discharges, emissions and losses [7]. Additionally, a few dyes and pigments were classified as potentially hazardous by the RICH-tool and as these primarily are used to beautify various personal care products and industrial cleaners it should be relatively easy to substitute these commodities to other commodities contain more eco-friendly colours or uncoloured ibidem. Further, mitigation options will be discussed with the industries and businesses in the close future, especially addressing the substances also listed as problematic by the Swedish Chemicals Agency [8]. 


\subsection{Discussion}

This study was hampered by incomplete data provided by the industries and businesses such as lack of CAS for various substances. Some industries reported which commodities that were used in manors that caused them to be discharged in to wastewater but not how much that actually were used, whereas others reported the consumption in volume or amount of all commodities used for operation and maintenance but failed to report whether the application generated wastewater discharges. Some industries omitted to provide information on chemical composition of their commodiites or were too vague in their replies, for example. merely reporting "anionic detergent".

Other obstacles in this type of studies are the lack of inherent data, primarily biodegradation, for the organic substances but even simple information such as the adsorption co-efficient $\left(\mathrm{K}_{\mathrm{oc}}\right)$ may be unavailable for certain types of substances, e.g., detergents and surface active agents.

\section{CONCLUSIONS}

The smalh and medium size industries contribute with a vast array of various substances to the municipal wastewater. Frequently used substances are pH-affecting substances such as inorganic acids and bases, organic solvents, oil \& grease, pesticides and preservatives. However, the majority of the studied organic substances did not pose a hazard to either the solid or the aqueous compartment. which is supported by the fact that most of the participating industries and businesses claim to use eco-labelled commodities intended for cleaning purposes. Questionnaires to industries and busimesses are an important way to retrieve information of the chemical composition of the municipal wastewater but it must be expected that fragmentary data can be submitted. Thus chemical analyses cannot be omitted for a full evaluation. Measures to reduce the presence of non-wanted substances in sludge can in some cases be easily implemented, based on the applied approach.

\section{ACKNOWLEDGMENTS}

The contributing industries and businesses in Eslöv municipality are highly acknowledged. This study would not have been possible without their contribution. Financial contribution from the ReVAQ-project is also acknowledged.

\section{REFERENCES}

[1] von der Heyden, C.J., Pegram G.C., Pema K., 2007. Non-point sources and the waste discharge charge system. Wat. Sci. \& Tech. 55(3), 45-54

[2] ReVAQ, 2007. Information available on their homepage http://www.revaq.se/ (2007-0707) (in Swedish)

[3] Baun A, Eriksson E, Ledin A, Mikkelsen PS., 2006 A methodology for screening for problem and hazard identification of xenobiotic organic compounds in stormwater. Sci. Total Environ. 370(1), 29-38

[4] Ledin, A., Eriksson, E., Baun, A., Aabling, T., Mikkelsen, P.S., 2006. CHIAT Chemical Hazard Identification and Assessment Tool En metodik för utvärdering av kemiska risker i samband med hantering av dag- och avloppsvatten, VA - Forsk rapport $\mathrm{Nr}$ 2005-09. Retrievable from http://www.dataforlaget.net/ vav/filer/VA-Forsk_200509.pdf (2007-09-15) (in Swedish) 
[5] US. Environmental Protection Agency, 2007. Estimation Program Interface (EPI) Suite (EPI 3.20), including PhysProp. Retrievable from http://www.epa.gov/opptintr/exposure/pubs/episuite.htm (2007-09-15)

[6] European Commission, 2001. Decision No 2455/2001/EC of the European parliament and the Council of 20 November 2001 establishing the list of priority substances in the field of water policy and amending Directive 2000/60/EC

[7] European Commission, 2000. Directive 2000/60/EC of the European Parliament and of the Council Establishing a Framework for the Community Action in the Field of Water Policy, 23 October 2000.

[8] Swedish Chemicals Agency. 2007. PRIO - a risk reduction tool of chemicals. Retrievable from http:/www.kemi.se/templates/PRIOEngframes 4144.aspx (200709-29)

[9] Garcia. M.T., Campos. E., Dalmau, M., Illan. P., Sanchez-Leal, J., 2006. Inhibition of biogas production by alkyl benzene sulfonates (LAS) in a screening test for anaerobic biodegradability. Biodegradation 17, 39-46

[10] Gejlsbjerg, B.. Klinge, C., Samsoee-Petersen, L., Madsen. T., 2001. Toxicity of linear alkylbenzene sulfonates and nonylphenol in sludge-amended soil. Environ. Toxicol. Chem. 20(12), 2709-2716.

[11] Elsgaard. L., Petersen. SO., Debosz, K., 2001. Effects and risk assessment of linear alkylbenzene sulfonates in agricultural soil. 2. Effects on soil microbiology as influenced by sewage sludge and incubation time. Environ. Toxicol. Chem. 20(8), 16641672. 